\title{
¿Puede el CCC-2 diferenciar perfiles pragmáticos?
}

\author{
¿Can the CCC-2 differentiate pragmatic profiles?
}

\section{Elvira Mendoza}

Departamento de Personalidad,

Evaluación y Tratamiento

Psicológico

Universidad de Granada, España

Marina Garzón

Departamento de Personalidad, Evaluación y Tratamiento

Psicológico

Universidad de Granada, España

\section{RESUMEN}

Objetivo: El presente trabajo pretende comprobar la capacidad de diferenciación y detección de perfiles pragmáticos de la traducción española de la Children's Communication Checklist-2 (CCC-2). Método: Se realizó el perfil normativo del instrumento en una muestra de 100 sujetos de habla española. Para determinar hasta qué punto la traducción española de la CCC-2 puede diferenciar entre niños con trastornos pragmáticos del lenguaje y niños con trastornos del lenguaje expresivo se realizó una comparación entre 10 sujetos con síndrome de Asperger y 10 sujetos con síndrome Down con un grupo Control. Resultados: La traducción española de la CCC-2 es capaz de identificar perfiles pragmáticos y de diferenciar entre perfiles normativos y clínicos como el del síndrome de Asperger y el del síndrome de Down. Además, este instrumento es capaz de obtener una imagen del perfil cuantitativo de las dificultades de comunicación de estos niños.

Palabras claves: lenguaje formal, pragmática, comunicación social, síndrome de Down, síndrome de Asperger.

\begin{abstract}
Objective: The current study aims to check the ability of the Spanish translation of the Children's Communication Checklist-2 (CCC-2) to discriminate and identify pragmatic profiles. Method: A normative profile of the instrument was performed on a 100 Spanish-speaking sample. To determinate to what extend the Spanish translation of the CCC- 2 is able to discriminate between children with a pragmatic language disorder and an expressive language disorder a comparison was made between 10 subjects with an Asperger syndrome and 10 subjects with Down syndrome versus a Control group. Results: The Spanish translation of the CCC-2 is able to identify pragmatic profiles and to discriminate between normative and clinic profiles as Asperger syndrome and Down syndrome. Furthermore, this instrument is able to obtain a quantitative profile picture of the communication impairments of those children.
\end{abstract}

Key words: formal language, pragmatics, social communication, Down Syndrome, Asperger Syndrome
Contacto con el autor: Elvira Mendoza Departamento de Personalidad, Evaluación y Tratamiento Psicológico Universidad de Granada, España España 


\section{¿Qué es la pragmática?}

Saber qué procesos se ponen en marcha en cada intercambio comunicativo nos ayuda a acercarnos a la raíz de las dificultades lingüísticas. Cuando estudiamos el estilo comunicativo de una persona debemos tener en cuenta varias dimensiones del lenguaje, como la fonológica, la sintáctica, la semántica o la pragmática. Estudiar la comunicación es, por tanto, estudiar aspectos muy diversos que van desde la fisiología hasta las relaciones sociales. La comunicación oral se relaciona tanto con las capacidades lingüísticas receptivas (decodificar el habla, reconocer palabras, interpretar o recordar una secuencia) y expresivas (seleccionar el mensaje apropiado, trasladar una idea a una frase o cambiar las formas de las palabras), como con la habilidad para integrar las palabras en el contexto y descubrir las intenciones de los hablantes ${ }^{1}$. Esta habilidad entra de lleno en el ámbito de la pragmática

La pragmática se ha estudiado desde distintas orientaciones teóricas. En la primera mitad del siglo XX, el semiótico C. W. Morris (1938) concibió el estudio de la Teoría de los Signos, o Semiótica, a partir de tres disciplinas: la sintaxis, la semántica y la pragmática. La sintaxis atendería a la relación formal entre un signo y otro, la semántica a los vínculos entre los signos y los objetos a que se refieren, mientras que la pragmática se ocuparía de la relación entre los signos y sus intérpretes. Así considerada, la pragmática abarca todos los fenómenos psicológicos, biológicos y sociológicos que tienen lugar en el funcionamiento de los signos. En otras palabras, la pragmática estudiaría todo lo relacionado con el uso de la lengua. A partir de los años 70, los lingüistas que se habían ocupado del estudio de los usos de la lengua intentaron delimitar el objeto de la pragmática y cimentar sus bases teóricas. En la actualidad, buena parte de esos investigadores consideran que la pragmática no es un componente de la teoría lingüística como pueda serlo la fonología, la morfología, la sintaxis o la semántica; tampoco pertenece a las disciplinas que relacionan el lenguaje con la realidad extralingüística, como la psicolingüística, la sociolingüística o la neurolingüística, sino que la pragmática constituye una perspectiva de estudio que puede ocuparse de cualquiera de estas disciplinas. La sintaxis, la semántica y la realidad extralingüística pueden considerarse bajo una perspectiva pragmática. Entendiendo la pragmática como la selección del mensaje o la interpretación adecuada en relación al contexto comunicativo ${ }^{1}$ podemos comprender cómo, desde una visión holística, la pragmática es una dimensión del lenguaje que actúa como interfaz entre las habilidades sociales, cognitivas $y$ lingüísticas ${ }^{2}$.

La dimensión pragmática del lenguaje es anterior a las demás, ya que está presente aun cuando no lo está el lenguaje oral ${ }^{3}$. Pensemos, por ejemplo, en un bebé y cómo éste influye sobre la conducta de la madre al llorar. Cuando un hablante emite un mensaje, sea verbal, no verbal o incluso preverbal (como hace el bebé), pretende ejercer una influencia en el otro, o conseguir unos objetivos específicos $^{4}$. Cuando se altera la pragmática se entorpece la comunicación social. De hecho, en la última revisión del DSM (DSM-5) ${ }^{5}$, que aparecerá en 
marzo de 2013, se propone el grupo de Trastornos de la Comunicación Social como subgrupo dentro de los Trastornos del Neurodesarrollo. Lo que comparten estos trastornos es la existencia de una discapacidad esencial en aspectos pragmáticos de formulación y comprensión de la comunicación social. Esta discapacidad afecta al desarrollo de las relaciones sociales y a la comprensión del discurso. Como el resto de trastornos, implica limitaciones funcionales: comunicación no efectiva, falta de participación social, rendimiento académico u ocupacional bajo, etc.

Las dificultades pragmáticas se disocian de las habilidades fonológicas o semántico-sintácticas en muchos $\operatorname{casos}^{6}$. Se han registrado casos en los que existen débiles habilidades pragmáticas a pesar de la presencia de una fonología, sintaxis y semántica casi intactas, del mismo modo que se han registrado casos de perfiles opuestos ${ }^{7}$.

El uso del contexto es vital para la adaptación y, por ende, para la supervivencia del ser humano. Los problemas de pragmática, por definición, dependen del contexto ${ }^{1}$. El empleo de las fórmulas de cortesía, por ejemplo, implica que el sujeto conoce el código a través del cual se lleva a cabo la comunicación, pero también la situación idónea para generalizar su uso ${ }^{8}$.

\section{Dificultades en la evaluación pragmática}

La primera alusión a la pragmática en el estudio de los trastornos del lenguaje no aparece hasta la década de los 70. Debido a esta corta trayectoria, hoy por hoy faltan herramientas, tanto teóricas como prácticas, para superar, identificar e incluso definir los déficit pragmáticos que se dan en algunos trastornos del lenguaje.

Adams, en una revisión de 2002, concluye que existen tres grandes obstáculos en la evaluación de la pragmática:

- Es compleja, ya que requiere comprobar y analizar conductas manifiestas, conductas que deberán inferirse y conductas que representan distintos niveles de procesamiento, lo cual dificulta notablemente la metodología de evaluación.

- La falta de un índice fiable de desarrollo normativo impide establecer comparaciones entre grupos o individuos.

- La existencia de influencias cognitivas, sociales y culturales en el uso del lenguaje. En definitiva, la influencia del contexto.

Otro obstáculo registrado en la evaluación de la dimensión pragmática es el solapamiento entre conductas lingüísticas y comunicativas propias de los trastornos específicos del lenguaje y de otros trastornos del desarrollo como el trastorno autista $^{7,9,10}$.

Bishop $^{11}$ (2003) publicó la segunda edición del Children Comunication Checklist (CCC-2), que sustituye al CCC, desarrollado previamente por la misma autora en $1998^{12}$. Se trata de un cuestionario diseñado para medir varios aspectos de la comunicación, cubriendo desde las habilidades de estructuración del lenguaje hasta las pragmáticas y de comunicación social. Ha sido estandarizado en una amplia muestra $(\mathrm{N}=542)$ de niños ingleses de 4 a 16 años con un desarrollo típico. Está diseñado para 
niños cuyo lenguaje no se limite a palabras aisladas, sino que construyan frases en sus emisiones lingüísticas. Sin embargo, no está pensado para niños de contextos culturales diferentes a los de colegios elementales de Reino Unido. Es útil en casos de niños con problemas del lenguaje ya identificados y para niños de otros grupos clínicos ${ }^{13}$. También puede discriminar entre niños con problemas clínicos de comunicación y niños con un desarrollo típico, así como diferenciar a niños con trastornos específicos del lenguaje de niños con discapacidad en la pragmática del lenguaje.

El CCC-2 contiene 10 escalas, cada una de las cuales se compone de 7 ítems. Es importante anotar que estos ítems no sólo indican déficits comunicativos (5 de 7) sino también fortalezas (2 de 7). En la Tabla 1 se especifica cada una de las escalas.

Tabla 1. Escalas e índices de la CCC-2.

\begin{tabular}{|c|c|c|}
\hline ESCALAS & INDICES & P.C. \\
\hline A. Habla & \multirow[t]{4}{*}{ Estructura y contenido lingüístico } & \multirow{2}{*}{$\begin{array}{l}\text { GCC } \\
\text { (puntuación general comunicativa) }\end{array}$} \\
\hline B. Sintaxis & & \\
\hline C. Semántica & & $\begin{array}{l}\text { SIDC } \\
\text { (problemas de interacción social) }\end{array}$ \\
\hline D. Coherencia & & \\
\hline E. Inicio inapropiado & \multirow[t]{4}{*}{ Pragmática } & \\
\hline $\begin{array}{ll}\text { F. } & \text { Lenguaje } \\
\text { estereotipado }\end{array}$ & & \\
\hline G. Uso del contexto & & \\
\hline $\begin{array}{l}\text { H. Comunicación no } \\
\text { verbal }\end{array}$ & & \\
\hline I. $\quad$ Relaciones sociales & \multirow[t]{2}{*}{ Comunicación social } & \\
\hline J. Intereses & & \\
\hline
\end{tabular}

El cuestionario permite extraer dos puntuaciones compuestas de las que se deriva interesante información. Con la puntuación compuesta de problemas de interacción social (SIDC) se puede identificar a aquellos niños con dificultades pragmáticas y sociales desproporcionadas en relación a su discapacidad en el lenguaje estructural. Lo que refleja esta puntuación es la diferencia que existe entre la suma de las escalas pragmáticas $E, H, I$ y J (puntuación compuesta de pragmática) y la suma de las escalas del índice de lenguaje estructural (A, B, C y
D). Por otro lado está la puntuación compuesta general comunicativa (CGC) que sirve para identificar niños con problemas significativos del lenguaje. Esta puntuación es el sumatorio de las ocho primeras escalas. Estas dos últimas puntuaciones compuestas son de gran utilidad a la hora de establecer perfiles de grupos clínicos. Cuenta, además, con una escala de consistencia entre los ítems formulados en negativo y en positivo. Si no se alcanza un mínimo en esta escala, el cuestionario debe ser desestimado. 
El CCC-2 provee de una buena medida de cribado de los déficits comunicativos y puede ser útil en la identificación de niños que necesitan de una evaluación más profunda en posibles TEA. Bishop y Baird $(2001)^{13}$ publicaron los datos de desarrollo pragmático típico en niños de 4 a 16 años obtenidos con la aplicación del CCC $^{12}$. La muestra, con una media de edad de 9.7 y una desviación típica de 3.5 años, permitió que se pudieran comparar niños con dificultades comunicativas con una muestra normativa.

El cuestionario puede ser cumplimentado por padres, familiares, educadores, logopedas y, en general, por cualquier persona que conozca y tenga contacto frecuente con el niño durante los tres meses anteriores como mínimo. Las respuestas se puntúan siguiendo una escala Likert. Los 50 primeros ítems están formulados en negativo: puntuación 0 (nunca realiza esta conducta) y 3 (siempre la realiza o más de dos veces al día). Los últimos 20 ítems se formulan en positivo: puntuación 0 (siempre la realiza o más de dos veces al día) y 3 (nunca la realiza).

Con esta investigación se pretende comprobar, por un lado, si el CCC-2, traducido y adaptado al español (Garzón, 2011), es una herramienta útil para realizar un cribado lingüístico y comunicativo de niños españoles con desarrollo típico del lenguaje y, por otro, si el CCC-2 discrimina entre los perfiles lingüísticos y comunicativos de niños cuya principal dificultad lingüística reside en el componente pragmático (Síndrome de Asperger) y de niños en los que las habilidades pragmáticas constituyen su principal fortaleza (Síndrome de Down).
Debido a la relevancia del contexto en el análisis de las habilidades pragmáticas es fácil entender cómo estudios con niños de habla inglesa residentes en Inglaterra, como los que se han venido haciendo hasta ahora, no cubren las demandas de detección y diferenciación de ciertos perfiles pragmáticos en otros contextos y lenguas. El hecho de desarrollar un instrumento diagnóstico en una cultura no garantiza su validez y fiabilidad en otras ${ }^{14}$. Hoy por hoy, este tipo de test o material de evaluación adaptado a población hispana es escaso o inexistente.

\section{Las poblaciones clínicas del estudio.}

En la clínica se usa el término Trastorno Generalizado del Desarrollo no Especificado (TGDNE) para referirse a un grupo de niños con una obvia discapacidad comunicativa pero que no presenta todas las características necesarias como para poder darle un diagnóstico de ninguno de los trastornos que se integran en los Trastornos Generalizados del Desarrollo (TGD). A partir de ahora se referirá a este grupo con el término de trastornos del espectro autista (TEA) que, si bien no figura en los sistemas actuales de clasificación, en el DSM-V sustituirá al de TGD. Tampoco está claro hasta qué punto el Trastorno Específico del Lenguaje (TEL), especialmente receptivo, y el TGD-No Especificado (NE) se solapan. De hecho, la descripción del TGD-NE ha sido considerada insatisfactoria, aunque no existen actualmente otras alternativas en uso.

$$
\text { Bishop y Norbury }(2002)^{7} \text {, utilizando }
$$
múltiples aproximaciones de evaluación estandarizada, encontraron que existe una gran 
proporción de niños con TEL que no encaja ni con las características del TEL ni con ninguno de los TEA.

Bishop y Baird $(2001)^{13}$ examinaron las habilidades funcionales de comunicación utilizando el Children Comunication Checklist (CCC) y encontraron diferencias entre los grupos clínicos. Fueron especialmente significativas entre los niños con trastornos específicos del aprendizaje y los niños con TEA. También encontraron importantes diferencias entre niños con el Síndrome de Asperger y niños con TGDNE. En contraposición con el grupo de TEA, el grupo de TGDNE no se diferenció del grupo de trastornos específicos del aprendizaje.

Entonces, además de la dificultad en la medición de la pragmática, nos encontramos con el debate de hasta qué punto niños con dificultades pragmáticas pertenecen a un subgrupo distinto del $\operatorname{TEA}^{15,16}$ o son sólo niños con autismo a los que se les ha dado una etiqueta diferente ${ }^{15,17}$.

\section{- Los Trastornos del Espectro Autista (TEA) y el Síndrome de Asperger (SA)}

La Prevalencia del TEA es de uno por cada 165 niños. Sólo un 25\% presenta discapacidad intelectual $^{18}$. El autismo es mucho más común de lo que se pensaba antiguamente y no está necesariamente asociado a una discapacidad cognitiva severa. En un extremo están los niños con autismo cuyo léxico y sintaxis se encuentran dentro de los límites de la normalidad ${ }^{19}$ y en otro, los niños con capacidades lingüísticas limitadas o incluso ausentes.

Allen y Rapin (1992) ponen el acento en el déficit lingüístico que presentan estos niños. Plantean que los niños autistas son en realidad disfásicos y que en ellos podemos encontrar todas las variantes de los diferentes tipos de trastornos específicos del lenguaje, con excepción de los puramente expresivos, ya que la comprensión nunca es totalmente adecuada. Incluso cuando el léxico y la sintaxis aparecen intactos, los déficits pragmáticos persisten. La pragmática se ha descrito como el dominio comunicativo que se encuentra persistentemente afectado en los TEA ${ }^{19,20,21}$.

Tradicionalmente, se ha utilizado el diagnóstico de síndrome de Asperger (SA) para referirse a un TEA combinado con buen lenguaje e inteligencia. Con buen lenguaje nos referimos a que la expresión no está alterada, aunque existen importantes déficits en la comunicación social. Las principales características comunicativas descritas para el síndrome de Asperger (Burgoine y Wing, 1983) son las siguientes: falta de empatía, interacción inapropiada e ingenua, poca o ninguna habilidad para hacer amigos, conversación pedante o repetitiva, pobre comunicación no verbal, absorción intensa en ciertos temas, lenguaje peculiar o pedante, dificultad para utilizar términos referidos a estados mentales, inadecuación pragmática de los enunciados, desconocimiento de pautas pragmáticas subyacentes en el diálogo, utilización inadecuada de pronombres, tendencia a la interpretación literal, dificultad para interpretar enunciados metafóricos o con doble sentido e incapacidad para comprender elementos de la comunicación no-verbal.

\section{- El Síndrome de Down}

En el Síndrome de Down el lenguaje oral se adquiere de forma natural y los primeros hitos del lenguaje aparecen con normalidad. Aunque los 
patrones lingüísticos corresponden a los de niños de menor edad cronológica, el desarrollo se produce de forma coherente con el desarrollo del nivel cognitivo.

La afectación lingüística es variable de unos individuos a otros, puesto que el $90 \%$ padece un compromiso mental importante y el $10 \%$ restante tiene un $\mathrm{Cl}$. límite. El área del lenguaje es la más retrasada en el síndrome, sobre todo, en aspectos expresivos. De hecho, numerosos estudios demuestran la existencia de un TEL- expresivo en el Síndrome Down ${ }^{22,23,24}$.

Los niños con SD, aun con un "correcto" desarrollo cognitivo, tienen problemas en el lenguaje, siendo los más frecuentes el retraso en la expresión oral y la tartamudez ${ }^{25}$. Tienen mayores dificultades en el lenguaje expresivo que en el comprensivo ${ }^{26}$. A medida que van creciendo, se abre la brecha entre la comprensión del lenguaje, que parece ser buena, y la producción, que es bastante deficitaria. La fluidez y el ritmo del lenguaje están muy afectados. Las dificultades expresivas pueden abarcar desde una simple pobreza de vocabulario, cuyas construcciones sintáctico-morfológicas sean correctas, hasta un lenguaje "autónomo". Existen casos en los que incluso no se llega a utilizar la expresión oral. Al igual que ocurre en el TEL- expresivo, la memoria verbal a corto plazo es muy deficitaria. Al estar las habilidades motoras orales comprometidas, el discurso de estos niños es poco inteligible y aparecen numerosos patrones atípicos del habla ${ }^{23}$.

Con respecto a la pragmática, los niños con SD manifiestan un desarrollo pragmático similar al de niños igualados en edad mental. A pesar de presentar algunas debilidades en el inicio y elaboración de temas conversacionales y en algunos aspectos de la narrativa, sus principales fortalezas se encuentran en una gran variedad de funciones comunicativas, como mantenimiento e inicio de temas conversacionales, respuestas a peticiones de clarificación y suficiente contenido narrativo en recontado cuando se apoyan en indicadores visuales ${ }^{27}$. Al compararlos con otros síndromes que conllevan retraso cognitivo, como el X-frágil, sus habilidades comunicativas y sociales son claramente superiores ${ }^{28}$.

Este trabajo se desarrolla en dos estudios, cada uno de los cuales intenta alcanzar uno de los objetivos propuestos. En el primero se intenta documentar si la traducción española del CCC-2 puede ser útil para realizar un cribado lingüístico y comunicativo de niños españoles con desarrollo típico del lenguaje. En el segundo se pretende comprobar si el CCC-2 discrimina entre los perfiles lingüísticos y comunicativos de niños cuya principal dificultad radica en el componente pragmático (Síndrome de Asperger -SA-) y de niños en los que las habilidades pragmáticas constituyen su principal fortaleza (Síndrome de Down -SD-).

\section{Objetivos e hipótesis de investigación.}

Esta investigación se divide en dos estudios, ya que se persiguen dos objetivos diferenciados:

Objetivo 1: Determinar el perfil normativo de la traducción española del CCC-2 en una muestra de niños de habla española. Se espera que el CCC-2 refleje un desarrollo armónico de las distintas dimensiones lingüísticas estudiadas, por lo cual el 
índice SIDC (diferencia entre la suma de las escalas pragmáticas $-E, H, I$ y J- y la suma de las escalas del índice de lenguaje estructural -A, B, C y D-) debe alcanzar valores relativamente bajos. Igualmente, en un desarrollo armónico deben registrarse correlaciones elevadas entre todas las variables.

Objetivo 2: Determinar si la traducción española de la CCC-2 puede diferenciar entre niños cuya alteración lingüística principal radica en el área pragmática (Síndrome de Asperger) y niños cuyo trastorno lingüístico principal afecta al lenguaje expresivo (Síndrome de Down). En ambos casos se espera que el índice CGC adquiera valores superiores $^{1}$ a los de la población típica. Con respecto al índice SIDC se espera que el grupo SA adquiera altas puntuaciones, mientras que el grupo SD debe obtener muy bajas puntuaciones 0 , incluso, negativas. $^{2}$

\section{Método}

\section{Estudio primero}

Participantes:

En este primer estudio participaron 100 niños con edades comprendidas entre 8 y 12 años. Todos los niños tenían un desarrollo lingüístico y académico normal y pertenecían a familias de clase media, dato estimado por los centros escolares a los que asistían. Se seleccionaron niños de este rango de edad y no del rango total al que el cuestionario es aplicable (de 4 a 16 años), porque el interés de esta

1 Es teste estudio no se han transformado las puntuaciones positivas y negativas. Las puntuaciones más altas indican una mayor alteración y las más bajas se pueden considerar normales

2 Por la misma razón anterior, ya que las puntuaciones más altas indican un mayor deterior investigación no es de tipo normativo (establecimiento de las puntuaciones mínimas y máximas de cada edad), por lo que seleccionamos un rango de edad en el que se espera estabilidad de las puntuaciones obtenidas en el CCC-2. En la Tabla 2 se muestra el número de sujetos por grupo de edad:

Tabla 2. Distribución de sujetos por grupo de edad

\begin{tabular}{lcccccc}
\hline $\begin{array}{l}\text { Grupo de } \\
\text { edad }\end{array}$ & $\mathbf{8}$ & $\mathbf{9}$ & $\mathbf{1 0}$ & $\mathbf{1 1}$ & $\mathbf{1 2}$ & TOTAL \\
\hline $\mathbf{N}$ & 23 & 21 & 24 & 17 & 15 & 100 \\
\hline
\end{tabular}

Procedimiento:

La traducción del CCC-2 implicó algunos cambios de la escala original. A pesar de que en la traducción se respetó al máximo la literalidad de los ítems, hubo que hacer modificaciones referidas a los usos de la lengua, a la morfología y a la sintaxis. Los ejemplos que se utilizan para aclararles a los informantes el significado de algunas preguntas han tenido que ser igualmente modificados.

Los informantes del cuestionario fueron padres/madres, tutores, educadores y/o logopedas. Como figura en las instrucciones de la escala original, puede actuar como informante cualquier adulto que haya tenido la oportunidad de interactuar con el niño durante un período mínimo de 3 meses. En algunos casos el envío del cuestionario se hizo por correo electrónico y fue devuelto por la misma vía. En otros casos se hizo de forma personal. Una vez recogidas las respuestas se pasaron a una base de datos y fueron analizadas estadísticamente con el paquete SPSS-15.

\section{Resultados}

Análisis de fiabilidad. Introduciendo las 10 escalas de la traducción española del CCC-2 se ha 
obtenido un alpha de Cronbach de 0.923. Se puede asumir, por tanto, una buena fiabilidad de la escala.

Diferencias debidas a la edad. Se realizó un análisis univariante considerando la puntuación total de la traducción española del CCC-2 como variable dependiente y la edad como factor de agrupación y no se han encontrado diferencias debidas a la edad. Se obtuvo el mismo resultado en los análisis de cada una de las variables. En la Tabla 3 se presentan los estadístico descriptivos de cada variable.

Tabla 3. Medias y desviaciones típicas de las variables del CCC- 2 y de los índices CGC y SIDC

\begin{tabular}{ccccc}
\hline ESCALA & Mínimo & Máximo $\left(^{*}\right)$ & Media & DT \\
\hline HABLA (H) & 0 & 12 & 1,54 & 2,249 \\
SINTAXIS (SINT) & 0 & 13 & 0,89 & 2,015 \\
SEMÁNTICA (SEM) & 0 & 13 & 2,40 & 2,613 \\
COHERENCIA (COH) & 0 & 13 & 1,90 & 2,452 \\
INIC- INADECUADA (I-IN) & 0 & 12 & $4,4,1$ & 3,052 \\
LENG. ESTEREOTIP. (L-EST) & 0 & 14 & 2,05 & 2,341 \\
USO CONTEXTO (CONT) & 0 & 17 & 2,41 & 2,861 \\
COM. NO VERBAL (CNV) & 0 & 14 & $\mathbf{2 , 2 2}$ & $\mathbf{2 , 7 1 4}$ \\
RELAC. SOCIALES (R-SOC) & 0 & 12 & $\mathbf{2 , 4 5}$ & $\mathbf{2 , 7 2 4}$ \\
INTERESES (INT) & 0 & 15 & 3,95 & 3,374 \\
CGC & 0 & 101 & $\mathbf{1 7 , 8 2}$ & $\mathbf{1 6 , 2 5 0}$ \\
SIDC & -5 & $\mathbf{6 0 , 3 0}$ & 6,41 \\
\hline TOTAL ESCALA & $\mathbf{0}$ & $\mathbf{1 2 4}$ & $\mathbf{2 0 , 4 7 4}$ \\
\hline
\end{tabular}

Se comprueba, por tanto, que en el rango de edad estudiado no existen diferencias debidas a la edad, por lo que no es necesario considerar el factor edad como covariable.

Al aplicar el cuestionario a una población típica, asumimos que las puntuaciones en todas las escalas deben estar correlacionadas, ya que al no existir dificultades específicas en ninguna dimensión lingüística, el desarrollo debe ser armónico. En la tabla 4 se presenta la matriz de las correlaciones de
Pearson entre las distintas escalas. En todas ellas la correlación ha resultado significativa $(p<0,0001)$.

\section{Discusión}

En primer lugar, podemos decir que se comprueba la estabilidad de la escala; una alpha de Cronbach de 0,93 explica la consistencia de la traducción española del CCC-2. La extendida propuesta de organización del lenguaje de Bloom y Lahey $^{29}$ en el ámbito educativo nos dice que debe entenderse éste como una intersección entre las tres vertientes (uso, forma y contenido). 
Tabla 4. Correlaciones entre escalas de la CCC-2

\begin{tabular}{|c|c|c|c|c|c|c|c|c|c|c|}
\hline & HABLA & SINTAX & SEMÁNT & COHEREN & $\begin{array}{l}\text { INIC- } \\
\text { INAD }\end{array}$ & $\begin{array}{l}\text { LENG- } \\
\text { ESTER }\end{array}$ & CONTEXT & $\begin{array}{l}\text { COM- } \\
\text { NO-V }\end{array}$ & $\begin{array}{l}\text { RELAC } \\
\text {-SOC }\end{array}$ & INTERESES \\
\hline HABLA & 1 & 0,742 & 0,552 & 0,779 & 0,356 & 0,645 & 0,606 & 0,583 & 0,484 & 0,376 \\
\hline SINTAXIS & 0,742 & 1 & 0,619 & 0,728 & 0,416 & 0,725 & 0,686 & 0,695 & 0,585 & \\
\hline SEMÁNTICA & 0,552 & 0,619 & 1 & 0,648 & 0,542 & 0,571 & 0,640 & 0,470 & & \\
\hline COHERENC. & 0,779 & 0,728 & 0,648 & 1 & 0,587 & 0,745 & 0,719 & & & \\
\hline INIC-INAD & 0,356 & 0,416 & 0,542 & 0,587 & 1 & 0,503 & & & & \\
\hline LENG-ESTER & 0,645 & 0,725 & 0,571 & 0,745 & 0,503 & & & & & \\
\hline CONTEXT & 0,606 & 0,686 & 0,640 & 0,719 & & & & & & \\
\hline COM-NO-V & 0,583 & 0,695 & 0,470 & & & & & & & \\
\hline RELAC-SOC & 0,484 & 0,585 & & & & & & & & \\
\hline INTERESES & 0,376 & & & & & & & & & \\
\hline
\end{tabular}

Se puede entender por intersección, en términos de Rapin y Allen (1978), el desarrollo armónico de las tres dimensiones del lenguaje, que en nuestra investigación hemos constatado a través de las altas correlaciones encontradas entre las distintas escalas del CCC-2.

Si analizamos la Tabla 3, podemos observar que las puntuaciones en las cuatro primeras escales (habla, sintaxis, semántica y coherencia), que aluden a las dimensiones de forma y contenido son inferiores $y$, por tanto, demuestran un rendimiento superior, que las pragmáticas y las de comunicación social. Esto explica que el valor promedio del índice SIDC $(6,30)$, sea positivo. Estos resultados siguen el mismo patrón que siguió la muestra sobre la que Bishop $(2003)^{11}$ baremó el CCC-2. Sin embargo, la diferencia encontrada entre las puntuaciones en cada escala no es significativa, lo cual indica una congruencia del estilo comunicacional y armonía en el desarrollo lingüístico de la muestra.

Pese a que Bishop encontró un mayor efecto suelo en las poblaciones de edades mayores, en nuestra muestra no se han encontrado diferencias. No obstante, debemos atender al hecho de que la muestra inglesa se compone de niños de 4 a 16 años, intervalo de edad para el cual está diseñado el CCC-2. Se podrían explicar las diferencias entre ambas muestras normativas por la diferencia entre los intervalos de edad de cada muestra, recordemos que nuestra muestra es de niños de 8 a 12 años.

\section{Estudio segundo}

Participantes:

En este segundo estudio participaron 30 niños y niñas de edades comprendidas entre los 8 y los 12 años procedentes de distintas instituciones 
clínicas y de asociaciones españolas. Los sujetos fueron divididos en tres grupos: Un primer grupo control de niños con desarrollo típico (grupo control -GC-) y dos grupos clínicos: niños con Síndrome de Asperger (SA) y niños con un Síndrome de Down (SD). Los sujetos de ambos grupos clínicos contaban con un diagnóstico previo. En este estudio no se tuvo en cuenta la distribución de edades en los grupos ya que, como se comprueba en el estudio 1, el rango de edad estudiado no afecta a las dimensiones lingüísticas analizadas.

\section{Procedimiento:}

La traducción española del CCC-2 fue completada por padres/madres, educadores y/o logopedas que tuvieran un contacto con los niños de una duración mínima de tres meses, tal como figura en las normas de aplicación de la versión original. En algunos casos el envío del cuestionario se hizo por correo electrónico y fue devuelto por la misma vía. En otros casos se hizo de forma personal. Una vez recogidas las respuestas se pasaron a una base de datos y fueron analizadas estadísticamente con el paquete SPSS-15. El diseño seguido ha sido quasiexperimental. Se utilizó un muestreo aleatorio para escoger a los componentes del grupo control extraídos de la población normativa española resultante del estudio 1 con el objetivo de realizar las comparaciones entre grupos.

Resultados:

Tras la realización del análisis de varianza univariante de cada escala se encontraron diferencias entre las medias obtenidas por cada grupo. En la Tabla 5 se representan los estadísticos descriptivos, junto con los valores de F, la probabilidad, el tamaño del efecto (eta cuadrado parcial) y las agrupaciones resultantes del análisis post-hoc de cada grupo en cada escala. Como se puede comprobar en la Tabla 5, existen diferencias entre los grupos en todas las escalas.

En un segundo análisis de subgrupos homogéneos específico para cada escala se establecieron las comparaciones entre los tres grupos y su nivel de significación. Se encontraron diferencias altamente significativas en todas las escalas. En la Tabla 4 se resumen las comparaciones entre los grupos para cada escala del CCC-2.

Como se puede ver en la tabla 5, al realizar los análisis post-hoc (DSH de Tukey) se observa que en las escalas de habla y sintaxis el grupo SA puntúa de forma similar al GC y en las escalas que analizan las relaciones sociales (relaciones sociales e intereses) es el grupo SD el que puntúa de forma similar al control. En las escalas de semántica y coherencia, aunque ambos grupos clínicos obtienen rendimientos inferiores a los del GC, el peor rendimiento lo tienen los niños con SD. En las escalas pragmáticas (iniciación inadecuada, lenguaje estereotipado, uso del contexto y comunicación no verbal) se invierte el modelo. Pese a la superioridad del grupo control, se aprecia un peor desempeño en el grupo SA que en el SD. 
Tabla 5. Comparaciones entre el grupo control y los grupos SA y SD. Estadísticos descriptivos, F, probabilidad y tamaño del efecto

\begin{tabular}{|c|c|c|c|c|c|c|}
\hline Escala & Grupo & MEDIA & DT & F (gl. 2) & $\begin{array}{c}\text { Eta cuadrado } \\
\text { parcial }\end{array}$ & Post-hoc \\
\hline \multirow[t]{3}{*}{ HABLA } & GC & 1,00 & 1,886 & $23,529 *$ & 0,635 & $\mathrm{CG}=\mathrm{SA}<\mathrm{SD}$ \\
\hline & SA & 2,30 & 2,497 & & & \\
\hline & SD & 11,20 & 5,432 & & & \\
\hline \multirow[t]{3}{*}{ SINTAXIS } & GC &, 10 & ,316 & $21,150^{*}$ & 0,610 & $\mathrm{CG}=\mathrm{SA}<\mathrm{SD}$ \\
\hline & SA & 3,10 & 2,685 & & & \\
\hline & SD & 9,50 & 5,039 & & & \\
\hline \multirow[t]{3}{*}{ SEMÁNTICA } & GC & 2,50 & 2,506 & $9,425^{*}$ & 0,411 & $\mathrm{CG}<\mathrm{SA}<\mathrm{SD}$ \\
\hline & SA & 6,00 & 1,633 & & & \\
\hline & SD & 7,60 & 3,565 & & & \\
\hline \multirow[t]{3}{*}{ COHERENCIA } & GC & 2,20 & 2,300 & $21,654^{*}$ & 0,616 & $\mathrm{CG}<\mathrm{SA}<\mathrm{SD}$ \\
\hline & SA & 9,40 & 2,716 & & & \\
\hline & SD & 9,50 & 3,408 & & & \\
\hline \multirow[t]{3}{*}{ INICIACIÓN INADECUADA } & GC & 3,60 & 3,373 & $17,131^{*}$ & 0,559 & $\mathrm{CG}<\mathrm{SD}<\mathrm{SA}$ \\
\hline & SA & 13,60 & 4,526 & & & \\
\hline & SD & 11,40 & 4,061 & & & \\
\hline \multirow{3}{*}{$\begin{array}{l}\text { LENGUAJE } \\
\text { ESTEREOTIPADO }\end{array}$} & GC & 1,50 & 1,354 & $27,430^{*}$ & 0,670 & $\mathrm{CG}<\mathrm{SD}<\mathrm{SA}$ \\
\hline & SA & 11,00 & 4,190 & & & \\
\hline & SD & 6,20 & 2,300 & & & \\
\hline \multirow[t]{3}{*}{ USO DEL CONTEXTO } & GC & 1,50 & 2,014 & $39,438^{*}$ & 0,745 & $\mathrm{CG}<\mathrm{SD}=\mathrm{SA}$ \\
\hline & SA & 12,10 & 3,143 & & & \\
\hline & SD & 9,70 & 3,093 & & & \\
\hline \multirow{3}{*}{$\begin{array}{l}\text { COMUNICACIÓN NO } \\
\text { VERBAL }\end{array}$} & GC & 1,70 & 1,889 & $33,838^{*}$ & 0,715 & $\mathrm{CG}<\mathrm{SD}<\mathrm{SA}$ \\
\hline & SA & 11,50 & 2,953 & & & \\
\hline & SD & 4,80 & 3,155 & & & \\
\hline \multirow[t]{3}{*}{ RELACIONES SOCIALES } & GC & 2,00 & 2,789 & $16,739 *$ & 0,554 & $\mathrm{CG}=\mathrm{SD}<\mathrm{SA}$ \\
\hline & SA & 10,90 & 3,814 & & & \\
\hline & SD & 5,60 & 3,688 & & & \\
\hline \multirow[t]{3}{*}{ INTERESES } & GC & 4,20 & 3,521 & $33,139 *$ & 0,711 & $C G=S D<S A$ \\
\hline & SA & 16,60 & 3,627 & & & \\
\hline & SD & 6,50 & 3,719 & & & \\
\hline
\end{tabular}

$\mathrm{P}<0,001$

Con el mismo procedimiento se analizaron los índices de la CCC-2 (GCC y SIDC). En la tabla 6 se pueden ver los estadísticos descriptivos de cada grupo y los resultados del análisis de varianza para cada agrupación.

Tabla 6. Resultados de la comparación entre grupos para cada índice de la CCC-2

\begin{tabular}{lcccccc}
\hline Escala & Grupo & MEDIA & DT & F & $\begin{array}{c}\text { Eta cuadrado } \\
\text { parcial }\end{array}$ & Post-hoc \\
\hline CGC & GC & 14,10 & 10,322 & $40,403^{*}$ & 0,750 & GC $<$ SA $=$ SD \\
& SA & 69,00 & 14,345 & $(\mathrm{gl}=2)$ & & \\
& SD & 69,90 & 21,121 & & & \\
\hline SIDC & GC & 5,70 & 6,816 & $31,197^{*}$ & 0,698 & SD $<$ CG $<$ SA \\
& SA & 31,80 & 11,535 & $(\mathrm{gl}=2)$ & & \\
& SD & $-9,50$ & 15,494 & & & \\
\hline
\end{tabular}

$P<0,001$ 
Como se observa en la tabla 6, en el índice de puntuación compuesta general comunicativa (GCC), los resultados son claramente superiores en el GC, con un importante tamaño del efecto. En el índice de puntuación compuesta de interacción social (SIDC), el grupo SD obtiene las puntuaciones más bajas, con un valor promedio negativo, lo que indica un rendimiento claramente inferior en las escalas de forma y contenido que en las pragmáticas, modelo opuesto al del grupo SA.

En la figura 1 se representan gráficamente las puntuaciones obtenidas por cada grupo en las distintas escalas y en la figura 2 las obtenidas en los índices GCC y SIDC, así como en las agrupaciones de las escalas lingüísticas (habla + sintaxis + semántica + coherencia), pragmáticas (iniciación inadecuada + lenguaje estereotipado + uso del contexto + comunicación no verbal) y sociales (relaciones sociales + intereses).

Tras comprobar cómo los grupos clínicos no siguieron un desarrollo armónico en sus perfiles comunicativos se realizó un tercer análisis para comprobar las correlaciones entre las escalas de la CCC-2 en los dos grupos experimentales (SA y SD). La Tabla 7 muestra las correlaciones existentes entre las escalas de la CCC-2 para los grupos experimentales (SA y SD).

En el análisis de correlaciones encontramos que las escalas de lenguaje estructural correlacionan entre sí. En la escala semántica existe además correlación significativa aunque más débil, con las escalas de iniciación inadecuada, lenguaje estereotipado y uso del contexto. La escala de coherencia no sólo correlaciona con las demás escalas de lenguaje sino con todas las de la CCC-2 de manera significativa.

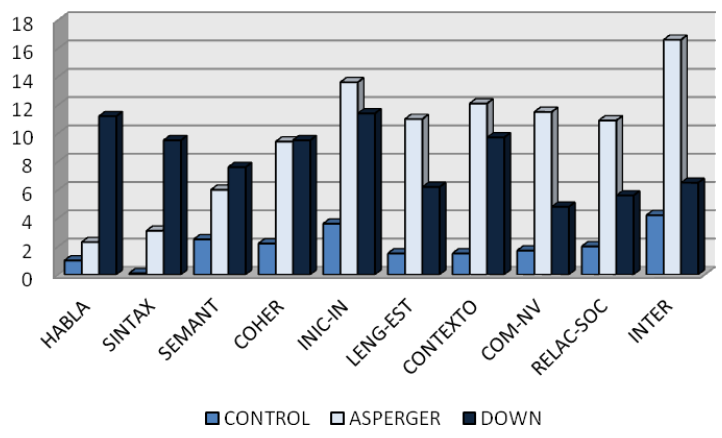

Figura 1. Representación gráfica de las puntuaciones obtenidas por cada grupo en las distintas escalas.

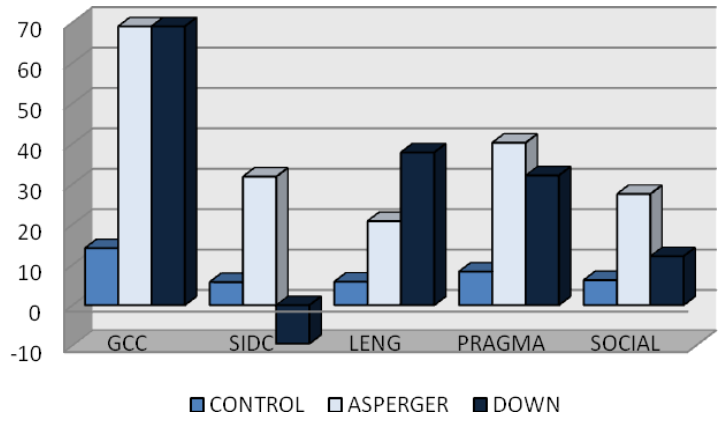

Figura 2. Representación gráfica de las puntuaciones obtenidas por cada grupo en índices CGC y SIDC y en las agrupaciones de puntuaciones (lenguaje, pragmática $y$ social).

En cuanto a las escalas pragmáticas decir que, efectivamente, existe una alta correlación entre ellas. La escala pragmática de uso del contexto demuestra una alta correlación con la escala de sintaxis. 
Tabla 7. Comparación de las correlaciones entre escalas de la CCC-2 en el GE1 y el GE2

\begin{tabular}{|c|c|c|c|c|c|c|c|c|c|}
\hline & HABLA & SINTAX & SEMÁNT & COHEREN & $\begin{array}{l}\text { INIC- } \\
\text { INAD }\end{array}$ & $\begin{array}{l}\text { LENG- } \\
\text { ESTER }\end{array}$ & CONTEXT & $\begin{array}{l}\text { COM- } \\
\text { NO-V }\end{array}$ & $\begin{array}{l}\text { RELAC- } \\
\text { SOC }\end{array}$ \\
\hline SINTAX &, $869\left(^{* * *}\right)$ & & & & & & & & \\
\hline SEMÁNT &, $631(* * *)$ & '707(***) & & & & & & & \\
\hline COHEREN &, $579(* *)$ &, $695\left(^{* *}\right)$ &, $637(* *)$ & & & & & & \\
\hline INIC-INAD & 280 &, $407\left(^{*}\right)$ & ,436(*) &, $729(* * *)$ & & & & & \\
\hline $\begin{array}{l}\text { LENG- } \\
\text { ESTER }\end{array}$ & 203 & 310 & ,369(*) &, $740(* * *)$ &, $748(* * *)$ & & & & \\
\hline CONTEXT & ,329 &, $466(* *)$ & ,452(*) &, $746(* * *)$ &, $754(* * *)$ &, $790(* * *)$ & & & \\
\hline COM-NO-V & ,068 & 165 & 346 &, $631(* * *)$ & $610(* * *)$ &, $849(* * *)$ &, $697(* * *)$ & & \\
\hline RELAC-SOC & 100 & 272 & ,309 &, $709(* * *)$ &, $731\left(^{* * *}\right)$ &, $843(* * *)$ &, $705(* * *)$ &, $798(* * *)$ & \\
\hline INTERESES &,- 133 & 010 & ,242 &, $534\left(^{* *}\right)$ & ,765(***) &, $794(* * *)$ &, $651(* * *)$ &, $756(* * *)$ &, $830(* *)$ \\
\hline
\end{tabular}

$* * * p<.001 ; * * p<.01 ; * p<.05$

\section{Discusión: estudio 2}

De los resultados del estudio 2 se plantea el debate sobre los perfiles lingüísticos que tiene cada grupo clínico y la capacidad de la traducción española de la CCC-2 para diferenciarlos. Por un lado, podemos establecer claras diferencias en las escalas entre los niños con SA y los niños con SD y la tendencia de cada grupo hacia un perfil lingüístico predominantemente formal o pragmático, respectivamente.

Podemos decir que los sujetos con síndrome de Asperger presentan mayor déficit en las escalas de iniciación inadecuada, lenguaje estereotipado, uso del contexto, comunicación no verbal, relaciones sociales e intereses, lo cual es de esperar teniendo en cuenta que los problemas del síndrome de Asperger son principalmente pragmáticos y sociales.

En cuanto a las fortalezas lingüísticas encontradas en los niños con síndrome de Asperger se menciona que las escalas relacionadas con el índice de lenguaje formal, como las de habla y sintaxis, alcanzan puntuaciones iguales a las de la población normal. Este resultado también es esperable, ya que los niños con síndrome de Asperger no tienen ningún tipo de trastorno formal del lenguaje.

Sin embargo, las puntuaciones obtenidas en este grupo en la escala semántica no llegan a igualarse a las del grupo control, aunque presentan significativamente un menor deterioro que los niños con síndrome Down. Este resultado se debe a que la escala semántica correlaciona, no sólo con el resto de escalas del índice de lenguaje estructural, sino también con algunas escalas del índice pragmático. Debemos entender que existe una interdependencia entre el rendimiento en semántica y ciertos parámetros de índole pragmática.

De igual manera ocurre con la escala de coherencia. El bajo rendimiento en coherencia del discurso de los niños con síndrome de Asperger se debe a que éste correlaciona, no sólo con el resto de escalas del índice de lenguaje estructural, sino también con el resto de escalas de la CCC-2. El hecho de que, de entre las escalas que conforman el índice de lenguaje estructural, ni la semántica ni la coherencia alcancen niveles de normalidad se debe a 
la influencia que las dimensiones pragmáticas y sociales del lenguaje ejercen sobre la forma de éste.

En el caso de los niños con síndrome Down los puntos fuertes se encuentran en escalas del índice social llegando a alcanzar niveles equiparables al grupo control tanto en la escala de relaciones sociales como en la de intereses. En cuanto a las escalas pragmáticas encontramos que, si bien los niños con síndrome Down no llegan a unos niveles normales, sí que superan a los niños con síndrome de Asperger, tanto en la escala de comunicación no verbal como en la de lenguaje estereotipado. Merecen especial reflexión las puntuaciones obtenidas en las escalas de iniciación inadecuada y uso del contexto ya que, tal y como ocurre en el caso de los Asperger, son escalas que dependen de otras. Como ha demostrado el análisis de correlaciones, el uso del contexto, pese a tratarse de una función pragmática, depende de los niveles en el resto de habilidades lingüísticas. En cuanto a la iniciación inadecuada en el grupo de niños con síndrome Down comprobamos cómo actúa de manera dependiente a la escala semántica ya que se han encontrado correlaciones entre ambas escalas. El deterioro del lenguaje estructural en estos niños explicaría los resultados obtenidos en la escala pragmática de iniciación inadecuada. En este sentido no podríamos decir que los niños con síndrome de Down tienen unas habilidades pragmáticas iguales a la población normativa, del mismo modo que ocurre con las habilidades lingüísticas formales en los niños con síndrome de Asperger.

En lo referente a los índices (GCC y SIDC), podemos decir que en el GCC, en el que se comparan los resultados obtenidos en todas las escalas menos en las de comunicación social, los dos grupos clínicos obtienen puntuaciones superiores y, por tanto, un rendimiento inferior, a las del grupo control, lo que indica la presencia de déficits lingüísticos y comunicativos en ambos grupos. Este resultado demuestra la capacidad de la traducción española de la CCC-2 para diferenciar grupos clínicos como el síndrome de Down o el de Asperger de niños con grupos de desarrollo típico del lenguaje.

De los resultados de la puntuación compuesta de problemas de interacción social (SIDC) se comprueba que la CCC-2 discrimina entre niños cuyo problema comunicativo principal es de tipo pragmático y social (SA) y niños con un trastorno lingüístico formal (SD). Claramente, en los niños con SD los problemas formales del lenguaje predominan sobre los pragmáticos y comunicativos, lo contrario de lo que sucede en los niños con SA. Este mismo resultado puede verse representado también en los índices de lenguaje estructural y pragmática, como ha quedado representado en la Figura 2.

\section{Discusión General}

Hemos podido comprobar cómo la traducción española de la CCC-2 es capaz de replicar los resultados obtenidos en la versión original de la CCC-2. Aunque el estilo comunicativo viene determinado con anterioridad a la adquisición del lenguaje oral, el desarrollo de las habilidades pragmáticas se produce como un proceso tardío de adquisición $^{30}$ que va desde los 4 años aproximadamente hasta la adolescencia. Esto explica 
unos niveles más bajos en las escalas pragmáticas en la muestra normativa, tanto de la escala original como de la versión traducida.

A pesar de lo reducido de nuestra muestra, el patrón de resultados es bastante similar al obtenido por Bishop (2003) ${ }^{11}$ en la estandarización de la CCC-2. Tal y como ocurre en la muestra australiana (compuesta por 111 niños), los valores quedan por debajo de la muestra normativa inglesa; es probable que suceda algo similar en la población de habla española, y que se pueda utilizar un punto de corte más bajo.

Los datos normativos que provee este estudio son muy útiles a la hora de identificar niños con retraso en el desarrollo de la comunicación. Sin embargo sería muy fructífero que estos estudios se realizaran en varias lenguas y culturas para indagar sobre una posible universalidad en el desarrollo del perfil comunicativo.

Hemos podido comprobar en el estudio 2 cómo la traducción española de la CCC-2 es una buena herramienta de cribado del desarrollo atípico del lenguaje; sin embargo la baremación de este instrumento al contexto del español es aún una asignatura pendiente para la investigación.

$$
\text { Los grupos clínicos difieren }
$$

significativamente del grupo control en la puntuación compuesta general comunicativa (GCC) y en prácticamente todas las escalas. En el caso de los niños con síndrome Down se igualan las puntuaciones en el índice social a las de la norma. Ocurre lo mismo en ciertas escalas del índice de lenguaje estructural en el síndrome de Asperger (habla y sintaxis). Por tanto, vemos cómo el perfil lingüístico resultante de los niños con síndrome Down es opuesto al perfil de los Asperger (véase la puntuación compuesta SIDC).

Aunque se esperaba que ambos grupos clínicos se diferenciaran aún más en sus perfiles comunicativos, la traducción española de la CCC-2 demuestra aquí su validez en la discriminación de los perfiles de ambos grupos experimentales, siendo capaz de diferenciar con precisión grupos con trastornos formales del lenguaje (SD) de grupos con trastornos predominantemente pragmáticos (SA).

En cuanto a los resultados relacionados con el síndrome de Asperger se puede decir que el desarrollo del lenguaje formal no es tan "normal" como se esperaba ya que no se alcanzan niveles de normalidad en todas las escalas. Como comprobó Howlin (2003), la mala ejecución en algunos tests de lenguaje de los niños con SA modifica la asunción de que el desarrollo del lenguaje es esencialmente normal en estos niños. En este sentido, se comprueba cómo el desarrollo semántico del lenguaje depende de las habilidades pragmáticas, a excepción, claro, de la comunicación no verbal. Recordemos que, el síndrome de Asperger dejará de ser una categoría diagnóstica en el DSM5 para integrarse dentro de los Trastornos del Espectro Autista (TEA), cuyo lenguaje se caracteriza por dificultades permanentes pragmáticas y sociales y dificultades variables en la estructuración del lenguaje.

En cuanto a los resultados de la escala de coherencia se ha comprobado que los niños con Síndrome de Asperger presentan grandes dificultades para ponerse en la piel del interlocutor y seguir las 
leyes de cooperación en el intercambio comunicativo. Es de esperar que la coherencia en el discurso esté alterada si, como en el caso del Síndrome de Asperger, también lo están las habilidades sociales y pragmáticas. Se ha encontrado este solapamiento entre conductas lingüísticas y comunicativas en diversos estudios anteriores en niños con TEL y otros trastornos del desarrollo como los TEA ${ }^{7,9,10}$.

Por otra parte, tras analizar los resultados obtenidos por el grupo de niños con Síndrome de Down en las escalas pragmáticas podemos decir que sus fortalezas lingüísticas radican principalmente en las habilidades sociales, al equipararse a las de la población normal. Es posible que sea esta ventaja la que los posiciona en un lugar preferente en relación al Síndrome de Asperger en cuanto a habilidades pragmáticas ya que, si la pragmática es una dimensión del lenguaje que actúa como interfaz entre las habilidades sociales, cognitivas y lingüísticas ${ }^{2}$, es de esperar que ésta sea mayor en el Síndrome de Down que en el de Asperger, ya que las deficiencias de este último grupo son, no sólo lingüísticas, sino también sociales.

Entonces, podríamos decir que la traducción española de la CCC-2 es capaz de identificar y diferenciar las fortalezas y debilidades de estos dos grupos clínicos.

La principal limitación de este trabajo fue el tamaño de la muestra. Aunque se ha comprobado que el rango de edad utilizado en este estudio no altera los resultados normativos, sería muy conveniente ampliar el rango de edad y analizar nuevamente el efecto de esta variable. Tampoco son las muestras de los grupos clínicos suficientemente grandes para sentar conclusiones, aunque son válidas experimentalmente para establecer las primeras aproximaciones. Convendría también aumentar el tamaño de estas muestras así como incluir otros Trastornos del Neurodesarrollo en el análisis.

Consideramos también que sería conveniente comparar las aplicaciones realizadas a padres, madres, logopedas y otros informantes. Aunque en la CCC-2 original no se establece diferenciación entre informantes, es probable que la percepción que tienen los padres de las fortalezas y debilidades de sus hijos pueda ser diferente de la que tienen los profesionales. Convendría comprobar el efecto de los informantes y, en su caso, establecer valores normativos diferenciados.

\section{Agradecimientos}

Agradecemos a la Asociación GranaDown su colaboración en el desarrollo de este estudio, así como a Juan Martos, al Centro Deletrea y a Gloria Sierra el acceso que nos han brindado a niños con Síndrome de Asperger. 


\section{Referencias}

1. Bishop, D. (1997). Uncommon understanding. Development and disorders of language comprehension in children. Hove, Psychology Press.

2. Adams, C. (2002). Practitioner Review: The assessment of language pragmatics. Journal of Child Psychology and Psychiatry, 43, 973-987.

3. Hallyday, M.A.K. (1975). Learning how to mean. Londres Arnold.

4. Mendoza, E. (2001). Trastorno Específico del Lenguaje. Ediciones Pirámide Psicología. Madrid 2010.

5. http://www.dsm5.org/ProposedRevision/Pages/Neuro developmentalDisorders.aspx

6. Tomblin, J. B., Zhang, X., Weiss, A., Catts, H., \& Ellis Weismer, S. (2004). Dimensions of individual differences in communication skills among primary grade children. In M. L. Rice \& S. F. Warren (Eds.), Developmental language disorders: From phenotypes to etiologies (pp. 53-76). Mahwah, NJ: Lawrence Erlbaum.

7. Bishop, D. V. M., \& Norbury, C. F. (2002). Exploring the borderlands of autistic disorder and specific language impairment: A study using standardized diagnostic instruments. Journal of Child Psychology \& Psychiatry \& Allied Disciplines, 43, 917-929.

8. Norbury C.F., Nash M., Bishop D.V.M. \& Baird G. (2004). Using parental checklists to identify diagnostic groups in children with communication impairment: A validation of the Children's Communication Checklist-2. International Journal of Language and Communication Disorders, 39, 345-364.

9. Shields, J. Varley, R. Broks, P. y Simpson, A. (1996). Hemispheric function in developmental language disorders and high-level autism. Developmental Medicine and Child Neurology, 38, 473-486.

10. Kjelgaard, M. M. \& Tager-Flusberg, H. (2001). An investigation of language impairment in autism: implications for genetic subgroups. Language and Cognitive Processes, 16, 287-308.
11. Bishop, D. V. M. (2003). The Children's Communication Checklist Second Edition (CCC-2). London: The Psychological Corporation.

12. Bishop, D.V.M. (1998). Development of children's communication checklist (CCC): A method for assessing qualitative aspects of communicative impairment in children. Journal of Child Psychology and Psychiatry, 39, 879-891.

13. Bishop, D. V. M. \& Baird, G. (2001). Parent and teacher report of pragmatic aspects of communication: Use of the Children's Communication Checklist in a clinical setting. Developmental Medicine and Child Neurology, $43,809-818$.

14. Andersen, W. Biringer, E. \& Helland, T. (2009). The usability of a Norwegian adaptation of the Children's Communication Checklist Second Edition (CCC-2) in differentiating between language impaired and nonlanguage impaired 6- to 12-year-olds. Scandinavian Journal of Psychology, 50, 287-292.

15. Brook, S. and Bowler, D. (1992). Autism by another name? Semantic and pragmatic impairments in children. Journal of Autism and Developmental Disorders, 22, 61-81.

16. Botting, N. (1998). Semantic-pragmatic disorder as a distinct diagnostic entity: Making sense of the boundaries (a comment on Boucher). International Journal of Language and Communication Disorders, 33, 71-108.

17. Gagnon, L., Mottron, L. Y Joanette, Y., (1997). Questioning the validity of the semantic-pragmatic syndrome diagnosis. Autism, 1, 37-55.

18. Chakrabarti, S. \& Fombonne, E. (2001). Pervasive developmental disorders in preschool children. Journal of the American Medical Association, 285, 3093-3099.

19. Landa, R. (2000). Social language use in Asperger syndrome and high-functioning autism. In A. Klin, F. Volkmar, \& S. Sparrow (Eds.), Asperger syndrome (pp. 125-158). New York, NY: Guilford Press.

20. Tager-Flusberg, H., Paul, R., \& Lord, C. (2005). Language and communication in autism. In F. Volkmar, R. Paul, A. Klin, \& D. Cohen (Eds.), Handbook of autism and pervasive developmental disorders (3rd ed., pp. 335364). Hoboken, NJ: Wiley. 
21. Young, E., Diehl, J., Morris, D., Hyman, S., \& Bennetto, L. (2005). The use of two language tests to identify pragmatic language problems in children with autism spectrum disorders. Language, Speech, and Hearing Services in Schools, 36, 62-72.

22. Fowler, A. (1990). Language abilities in children with Down syndrome: Evidence for a specific syntactic delay. In D.Cicchetti and M.Beeghly (Eds.), Children with Down syndrome: A developmental perspective. 9. (pp. 302328). New York, USA: Cambridge University Press 72.

23. Stoel-Gammon, C. (1997). Phonological development in Down syndrome. Mental Retardation and Developmental Disabilities Research Reviews, 3, 300-306.

24. Chapman, R. S. (1997). Language development in children and adolescents with Down syndrome. Mental Retardation and Developmental Disabilities Research Reviews, 3, 307-312.

25. Santos, E. y Bajo, C. (2011). Alteraciones en el lenguaje de los pacientes con síndrome de Down. Revista de la Sociedad Otorrinolaringológica de Castilla y León, Cantabria y La Rioja. Volumen 2, № 9.

26. Miller, J.F. (2001). Síndrome de Down: comunicación, lenguaje, habla. Masson. Barcelona, 2001.

27. Martin, G.E., Kusek, J., Estigarribia, B., \& Roberts, JE. (2009). Language characteristics on individual with Down Syndrome. Topics in Language Disorders, 29, 112-132.

28. Abbeduto, L., \& Murphy, M.M. (2004). Language, social cognition, maladaptative behavior, and communication in Down Syndrome and fragile $X$ Syndrome. En M.L. Rice y S.F. Warren (Eds.). Developmental language disorders. From phenothipes to etiologies. Mahwah, NJ: Erlbaum.

29. Bloom, L. and Lahey, M. (1978). Language Development and Language Disorders. Chapters 1, 2, 4, John Wiley and Sons, New York.

30. Bavin, E.L. (2009). Perspective on child language. In E.L. Bavin (Ed.) Handbook of Child Language (1-12). Cambridge University Press. 